\title{
Treatment of Capsular Contracture after Breast Augmentation with Serial Fat Grafting and Implantation
}

Tae Kwang Jeong', Jin Woo Han², Kyung Hee Min ${ }^{2}$

${ }^{1}$ HB Plastic Surgery Clinic, Seoul; ${ }^{2}$ Department of Plastic and Reconstructive Surgery, Eulji General Hospital, Eulji University School of Medicine, Seoul, Korea
This article was presented at the Aesthetic Plastic Surgery 2017 - Korean Society for Aesthetic Plastic Surgery (KSAPS) on April 1-2, 2017 in Seoul, Korea.

No potential conflict of interest relevant to this article was reported.
Background Capsular contracture is the most common complication after breast augmentation using implants. In case of severe capsular contracture, surgical correction is inevitable. We introduce a treatment for severe capsular contracture with serial fat grafting before augmentation using implants.

Methods In severe cases of capsular contracture, we removed the implants and performed partial capsulectomy. We harvested fat from the flanks or thighs using liposuction. The first fat graft was performed in the subdermal, subcutaneous, and subglandular layers of the breast. The harvested fat was frozen and stored. One month after implant removal and the first graft, a second fat graft was performed. One month later, a third fat graft was performed. Six months after implant removal, we augmented the breasts with implants.

Results The cases of severe breast contracture were treated without any complications. The volume and contour of the breasts were satisfactory.

Conclusions After implant removal in cases of severe capsular contracture, breast augmentation may be needed. Serial fat grafting before augmentation using implants prevents scarring of the breast envelope and provides sufficient volume with a secure breast envelope.

Keywords Breast, Implant capsular contracture, Transplantation autologous

\section{INTRODUCTION}

Aesthetic or reconstructive surgical procedures using breast implants are routinely performed, and in increasing numbers [1]. Capsular contracture is the most common complication after breast implant surgery, with reported incidence rates ranging from $2.8 \%$

Received: Jan 18, 2018 Revised: Mar 22, 2018 Accepted: Apr 11, 2018 Correspondence: Kyung Hee Min Department of Plastic and Reconstructive Surgery, Eulji General Hospital, Eulji University School of Medicine, 68 Hangeulbiseok-ro, Nowon-gu, Seoul 01830, Korea.

E-mail: mkh797@hanmail.net

Copyright @ 2018 The Korean Society for Aesthetic Plastic Surgery.

This is an Open Access article distributed under the terms of the Creative Commons Attribution Non-Commercial License (http://creativecommons.org/licenses/by-nc/4.0/) which permits unrestricted non-commercial use, distribution, and reproduction in any medium, provided the original work is properly cited. $\quad w w w . e-a a p s . o r g$ to $20.4 \%[2,3]$. Capsular contracture is caused by an excessive fibrotic reaction in the tissue surrounding the implant and is thought to be an inflammatory response [2]. The precise etiology and pathogenesis of capsular contracture remain unclear, although smooth implants and subglandular position are risk factors [4,5]. Capsular contracture can lead to deformed or painful breasts. In symptomatic capsular contracture (Baker grade III and IV), surgical treatment is needed. Capsulectomy with or without capsulotomy is routinely performed [4]. However, the rate of contracture recurrence after surgical treatment has been found to be $25 \%$, with most cases occurring within 5 years [6]. Therefore, various procedures have been performed to prevent recurrence. Autologous fat transfer and the use of acellular dermal matrix have been reported for the treatment of capsular contracture $[7,8]$. We present a novel and effective treatment using serial fat grafting before augmentation with implants. 


\section{METHODS}

Our treatment was performed in patients diagnosed with severe capsular contracture (Baker grade III and IV). Under general anesthesia, the breast implants were removed via an inframammary fold incision. Partial capsulectomy was then performed. The anterior capsule was completely removed, and open capsulotomy was performed with multiple incisions at the posterior capsule. After copious irrigation with antibiotic solution $(500 \mathrm{~mL}$ of saline, mixed with $1 \mathrm{~g}$ of cefazolin and $80 \mathrm{mg}$ of gentamicin), the incision site was closed layer by layer after drain insertion. Fat was harvested from the flank or thigh with a conventional liposuction cannula measuring $3 \mathrm{~mm}$ in diameter, under low pressure. The harvested fat was centrifuged at 3,000 rpm for 3 minutes. The purified fat was grafted through the periareolar and axillary incision sites with a 17-gauge cannula measuring 15 to $23 \mathrm{~cm}$ in length. Fat was grafted in small aliquots in the subdermal, subcutaneous, and subglandular planes to prevent fat necrosis. The injection plane, injection site, and fat volume were determined by the thickness and circulation status of the breast envelope. The remaining fat was frozen for storage at $-20^{\circ} \mathrm{C}$. Following fat grafting, a mild compressive dressing was performed using a cotton roll and elastic bandage. One month
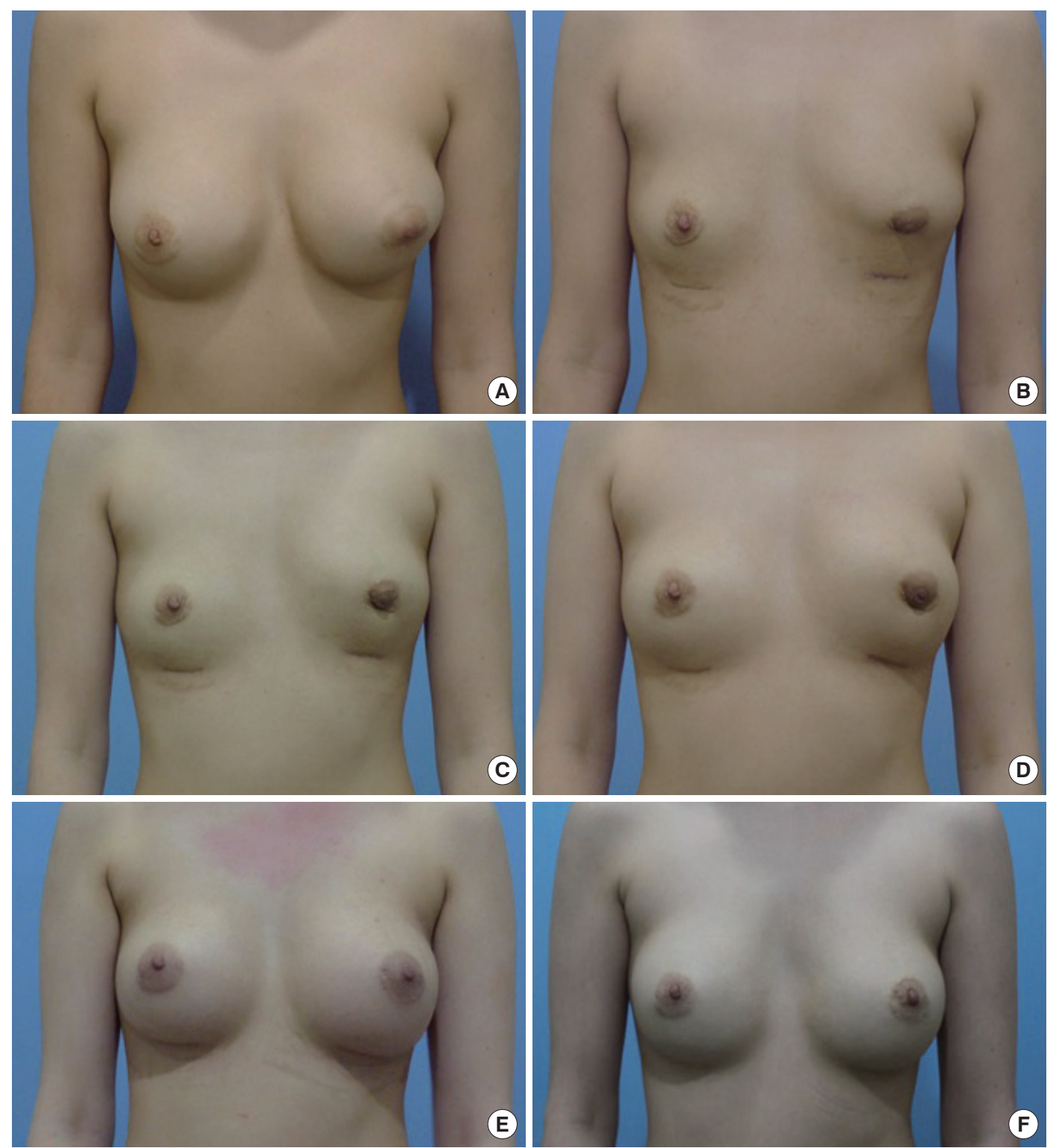

Fig. 1. (A) Preoperative view, Baker grade III (right) and IV (left). (B) Postoperative view 1 month after implant removal and the first fat graft. (C) Postoperative view 1 month after the second fat graft. (D) Postoperative view 1 month after the third fat graft. (E) Postoperative view at 1 month and (F) 19 months after augmentation with breast implants. 
after the first operation, a second fat graft was performed with the stored fat using the same surgical procedure. One month later, the third fat graft was performed. Six months after the first operation, breast augmentation with implants was performed via the previous inframammary incisions, along the same plane of insertion.

\section{RESULTS}

With the progression of fat grafting, we observed thickening and softening of the breast envelope. The irregular surface after capsulectomy and breast asymmetry was corrected through serial fat grafting. No scarring or fibrosis of the breast envelope after capsulectomy was detected. No other significant complications were found. The volume and contour of the breasts were satisfactory to the surgeons and the patients alike.

\section{Case}

A 33-year-old woman with capsular contracture classified as Baker grade III (right) and grade IV (left) presented with a history of augmentation mammoplasty through a periareolar incision with round textured breast implants (right, $280 \mathrm{~mL}$; left, $240 \mathrm{~mL}$ ) performed 5 years ago. After implant removal, $69 \mathrm{~mL}$ (right) and $84 \mathrm{~mL}$ (right) of fat was initially grafted. A month later, a second fat graft with 45 $\mathrm{mL}$ (right) and $78 \mathrm{~mL}$ (left) of fat was performed. After another month, a third graft was performed using $48 \mathrm{~mL}$ (right) and $72 \mathrm{~mL}$ (left) of fat. Six months after implant removal, the breasts were augmented with round textured breast implants (right, $220 \mathrm{~mL}$; left, $220 \mathrm{~mL}$ ) through an inframammary incision. During the followup period, which extended for almost 19 months postoperatively, no recurrence of capsular contracture was observed, resulting in a satisfactory aesthetic outcome (Fig. 1).

\section{DISCUSSION}

In 1912, Holländer [9] first described fat injection into the breast. However, in 1987, the American Society of Plastic Surgeon (ASPS) condemned the procedure because of concerns that it could interfere with the diagnosis of breast cancer [10]. Nonetheless, fat grafts into the breast have been performed in clinical settings. Coleman and Saboeiro [11] reported positive outcomes of fat grafting into the breast. Recently, several studies reported that fat grafting did not interfere with mammographic interpretation $[12,13]$. The ASPS stated in 2009 that "fat grafting may be considered for breast augmentation and correction of defects associated with medical conditions and previous breast surgeries; however, results are dependent on the technique and surgical expertise" [10]. Its clinical indications include breast augmentation, reconstruction after breast cancer surgery or radiotherapy-induced breast tissue damage, and the correction of breast deformities [10]. In addition, fat grafting is indicated for the treatment of capsular contracture, as well as breast augmentation with or without implants. A recent porcine model suggested that fat grafting may be used to treat capsular contracture by promoting neovascularization in the adjacent tissue and softening of the capsule [7]. Consistent with our method, Salgarello et al. [14] reported that fat grafting in irradiated breast tissue before implantation reduced radiation-related complications. Panettiere et al. [15] reported that serial fat grafting improved the outcomes of irradiated breasts that were reconstructed with implants. In one case, Baker grade III and IV contracture was downgraded to Baker grade I contracture after a single fat graft.

These therapeutic effects of fat grafting have been attributed to the regenerative effect of adipose-derived stem cells contained in the fat tissue. Adipose-derived stem cells secrete various cytokines, including angiogenic factors, which induce angiogenesis and wound healing $[7,14,15]$.

Our findings suggest that thinning and fibrotic changes after capsulectomy were altered by the treatment. Serial fat grafting thickened and softened the breast envelope. Fat grafting itself augmented breasts, suggesting that smaller breast implants could be used. Therefore, our method prevented thinning of the breast envelope and recurrence of capsular contracture following breast augmentation using implants. In addition, fat grafting corrected surface irregularities and breast asymmetry after capsulectomy. The grafting procedure was conducted 3 times at 1-month intervals due to the limited space available following fibrosis after capsulectomy. A large volume of fat may increase the risk of necrosis due to poor vascularization. Further, the varying conditions of the breast envelope in patients require evaluation at monthly intervals in order to ensure the safety and effectiveness of the serial interventions.

Nonetheless, when using frozen stored fat, it is important to consider the possibility of reduced viability and infection resulting from the freezing and thawing process.

Our results show that serial fat grafting before augmentation with implants in cases of severe capsular contracture resulted in satisfactory functional and aesthetic outcomes, without implantrelated complications. Our method represents an attractive treatment modality for cases of capsular contracture.

\section{PATIENT CONSENT}

Patient provided written consent for the use of her images.

\section{REFERENCES}

1. Singh N, Picha GJ, Hardas B, et al. Five-year safety data for more than 55,000 subjects following breast implantation: comparison of rare adverse event rates with silicone implants versus national norms and saline implants. Plast Reconstr Surg 2017;140:666-79.

2. Headon H, Kasem A, Mokbel K. Capsular contracture after breast augmentation: an update for clinical practice. Arch Plast Surg 2015;42: 
532-43.

3. Spear SL, Murphy DK. Natrelle round silicone breast implants: Core Study results at 10 years. Plast Reconstr Surg 2014;133:1354-61.

4. Namnoum JD, Largent J, Kaplan HM, et al. Primary breast augmentation clinical trial outcomes stratified by surgical incision, anatomical placement and implant device type. J Plast Reconstr Aesthet Surg 2013; 66:1165-72.

5. Stevens WG, Nahabedian MY, Calobrace MB, et al. Risk factor analysis for capsular contracture: a 5-year Sientra study analysis using round, smooth, and textured implants for breast augmentation. Plast Reconstr Surg 2013;132:1115-23.

6. Forster NA, Kunzi W, Giovanoli P. The reoperation cascade after breast augmentation with implants: what the patient needs to know. J Plast Reconstr Aesthet Surg 2013;66:313-22.

7. Roca GB, Graf R, da Silva Freitas R, et al. Autologous fat grafting for treatment of breast implant capsular contracture: a study in pigs. Aesthet Surg J 2014;34:769-75.

8. Cheng A, Lakhiani C, Saint-Cyr M. Treatment of capsular contracture using complete implant coverage by acellular dermal matrix: a novel technique. Plast Reconstr Surg 2013;132:519-29.

9. Holländer E. Die kosmetische Chirurgie. In: Leipzig MJ, editor. Handbuch der Kosmetik. Leipzig, DE: Veit \& Comp; 1912. p. 667-712.

10. Coleman SR, Saboeiro AP. Primary breast augmentation with fat grafting. Clin Plast Surg 2015;42:301-6, vii.

11. Coleman SR, Saboeiro AP. Fat grafting to the breast revisited: safety and efficacy. Plast Reconstr Surg 2007;119:775-85.

12. Veber M, Tourasse C, Toussoun G, et al. Radiographic findings after breast augmentation by autologous fat transfer. Plast Reconstr Surg 2011;127:1289-99.

13. Cameron JA, Auclair E, Nelson M, et al. Radiologic evaluation of women following cosmetic breast augmentation with silicone implants and fat grafting. Plast Reconstr Surg 2014;134 Suppl 4:91-2.

14. Salgarello M, Visconti G, Farallo E. Autologous fat graft in radiated tissue prior to alloplastic reconstruction of the breast: report of two cases. Aesthetic Plast Surg 2010;34:5-10.

15. Panettiere P, Marchetti L, Accorsi D. The serial free fat transfer in irradiated prosthetic breast reconstructions. Aesthetic Plast Surg 2009;33: 695-700. 